\title{
A Non-Orthogonal SVD-based Decomposition for Phase Invariant Error-Related Potential Estimation
}

\author{
Ronald Phlypo, Nisrine Jrad, Sandra Rousseau and Marco Congedo
}

\begin{abstract}
The estimation of the Error Related Potential from a set of trials is a challenging problem. Indeed, the Error Related Potential is of low amplitude compared to the ongoing electroencephalographic activity. In addition, simple summing over the different trials is prone to errors, since the waveform does not appear at an exact latency with respect to the trigger. In this work, we propose a method to cope with the discrepancy of these latencies of the Error Related Potential waveform and offer a framework in which the estimation of the Error Related Potential waveform reduces to a simple Singular Value Decomposition of an analytic waveform representation of the observed signal. The followed approach is promising, since we are able to explain a higher portion of the variance of the observed signal with fewer components in the expansion.

Index Terms-Hilbert transform, Singular Value Decomposition (SVD), phase plane, analytic signal, Error Related Potential (ErrP)
\end{abstract}

\section{INTRODUCTION}

The estimation of a single waveform from a mixture of this waveform and (noisy) background activity is a challenging task with impact in a wide range of applications. When the waveform is triggered by an external stimulus and multiple observations are available, the waveform can be distinguished from the ongoing background activity thanks to the property of time locking. Under the hypothesis that the background activity does not result from an interaction with the stimulus, the former guarantees that summing over the different observations marginalizes over the background activity and thus uncovers the waveform. In this work we will focus on these so-called phase locked waveforms in the specific case of Event-Related Potentials.

Event-Related Potentials (ERP) are local potential fields that emerge from the solicitation of a cortical neural population in order to disambiguate the interpretation of an observed event, generally called the trigger or stimulus. The ERP, as a response to these stimuli, is considered to be composed of half waves (i.e. negative or positive half periods of sinusoidal like signals) each having a specific temporal latency with respect to the stimulus onset. However, in practice we often do not observe these wave forms at exact latencies but rather at small variations around a mean latency value. The latter phenomenon gains in importance when the stimulus response elicits higher level cognitive processing. This is the case in the Error Related Potential (ErrP) based on a feedback of the result to the participant. The feedback

\footnotetext{
The authors are with the Vision and Brain Signal Processing Research Group at GIPSA-lab, Universities of Grenoble/CNRS UMR 5216 , Grenoble, FRANCE firstname. lastname@gipsa-lab.grenoble-inp.fr
}

indicates whether the participant answered correctly in a preceding cognitive task, e.g., memorization. If the feedback is in conflict with the participant's expectation, transient changes (half waves) in the potential field of the electroencephalogram (EEG) may be observed. It is suggested that in case the feedback requires cognitive processing (comparison between the expected outcome and the feedback), latencies as well as their inter-trial variabilities augment.

Since the ErrPs are not observed in isolation but in the presence of ongoing background activity, a straightforward processing of the observations is to align multiple trials with respect to the trigger onset and taking a (weighted) sum over the trials. By summing over different trials, the ErrP waveform is reinforced whilst the background activity - uncorrelated with the trigger onset - asymptotically approaches its (supposed) zero-mean value. Whilst this method is by far out the simplest approach to the estimation of the ErrP waveform, it is prone to errors inherent to the summing operation. Firstly, the sum is taken over all trials, without distinguishing between informative and non-informative trials. This default could be overcome by choosing optimal weights, e.g., as a function of the trial's signal-to-noise ratio [1]. Secondly, and more important, the summing suffers from the intertrial variabilities of the latencies over different trials [2]. Indeed, it can be shown that simple summing introduces non-negligible errors, especially when the number of trials is relatively low [3], [4].

In this work, we opt for a representation of the signal as a waveform. We allow for transformations of the waveform such as rotation in the phase plane and amplitude scaling [5] rather than time shift and stretch [4]. This offers considerable benefits in that we have a linear transformation. In addition, we empirically show that the variance of the observations is captured in less components with respect to a plain principal component analysis.

\section{METHODS}

\section{Notational Conventions}

All signals considered are supposed to be continuous in time. When calculations are performed, they are taken over a time limited window after being sampled. We will not, generally, explicit this sampling process in our calculations and suppose that the effect of sampling can be neglected. This is the case for ErrP waveforms since their frequency band of interest is band limited $(3-8 \mathrm{~Hz})$ with an upper frequency generally far below half the sampling frequency (typically 250,500 or $1000 \mathrm{~Hz}$ ). 
We will denote by $\Re$ and $\Im$ the real part, respectively the imaginary part of a complex number or vector. A column vector will be denoted as $\mathbf{x}=\left(x_{1}, x_{2}, \ldots x_{N}\right)^{T}$ and a matrix constructed from temporal samples of a vector signal as $\mathbf{X}=$ $\{\mathbf{x}(t)\}_{t}$. All other notations should be clear from the context.

\section{A. Analytic Waveform Representation of a Signal}

The Hilbert transform is used here to obtain the analytic waveform representation of a signal. To obtain the Hilbert transform $[\mathcal{H}(x)](t)$ of a continuous real time signal $x(t) \in$ $\mathbb{R}$, one convolves $x(t)$ with the non-causal infinite impulse response filter $h(t)=(\pi t)^{-1}$. This filter is not integrable and one may use the Cauchy Principal Value $(P V)$ However, in practice, we will make use of the following identities:

$$
\forall f_{p}:\left\{\begin{array}{rrr}
{[\mathcal{H}(\cos )]\left(2 \pi f_{p} t+\phi\right)} & = & \sin \left(2 \pi f_{p} t+\phi\right) \\
{[\mathcal{H}(\sin )]\left(2 \pi f_{p} t+\phi\right)} & = & -\cos \left(2 \pi f_{p} t+\phi\right)
\end{array}\right.
$$

Suppose that the signal can be arbitrarily well approximated by its Fourier series expansion ${ }^{1}$

$$
x(t)=\sum_{p} a_{p} \cos \left(2 \pi f_{p} t\right)+b_{p} \sin \left(2 \pi f_{p} t\right),
$$

then - since the Hilbert transform is defined by a linear operator - it follows that $[\mathcal{H}(x)](t)=\sum_{p} a_{p} \sin \left(2 \pi f_{p} t\right)-$ $b_{p} \cos \left(2 \pi f_{p} t\right)$ and thus the analytic signal

$$
\begin{aligned}
\tilde{x}(t) & =x(t)+\imath[\mathcal{H}(x)](t) \\
& =\sum_{p}\left(a_{p}-\imath b_{p}\right)\left[\cos \left(2 \pi f_{p} t\right)+\imath \sin \left(2 \pi f_{p} t\right)\right] .
\end{aligned}
$$

For a limited time signal, $a_{p}$ and $b_{p}$ can be obtained from its discrete Fourier transform, since $[\mathcal{F}(x)]\left(f_{p}\right)=\frac{1}{2}\left(a_{p}-\right.$ $\left.\imath b_{p}\right), \forall f_{p}>0$, and $[\mathcal{F}(x)](0)=a_{0},\left(b_{0}=0\right)$. We thus obtain the analytic waveform from the Fourier transform $[\mathcal{F}(x)](t)$ of $x(t)$ by considering

$$
[\mathcal{F}(\tilde{x})](f)= \begin{cases}0 & , \forall f<0, \\ {[\mathcal{F}(x)](f)} & , f=0 \\ 2 \times[\mathcal{F}(x)](f) & , \forall 0<f \leq F_{s} / 2 .\end{cases}
$$

where $F_{s}$ is the sampling frequency.

Back in the time domain we find that the real part of our analytic waveform representation $\Re\{\tilde{x}\}(t)$ is precisely the initially observed signal $x(t)$. We may also identify $\psi(t)=\tan ^{-1} \frac{\Im\{\tilde{x}\}(t)}{\Re\{\tilde{x}\}(t)}$ with the instantaneous phase and $\omega(t)=\partial \psi(t) / \partial t$ with the instantaneous frequency of the waveform. In other words, we may think of the analytic signal representation as a $\left(\right.$ global $\left.^{2}\right)$ time-frequency representation of our signal. A local signal representation of the signal $x(t)$ as a waveform would thus look like $\tilde{x}(t)=|\tilde{x}(t)| e^{\imath \psi(t)}=|\tilde{x}(t)| e^{\imath(2 \pi f(t) t-\phi(t))}$, where $|\tilde{x}(t)|$ is also called the instantaneous amplitude or envelope of the waveform.

\footnotetext{
${ }^{1}$ For a sampled signal this approximation is exact if the frequencies $f_{p}$ are chosen as $p F_{s} / P$ for $p \in\{0,1,2, \ldots, P-1\}$, where $P$ are the number of available samples. In many cases the number of frequencies can be considerably reduced without compromising this approximation.

${ }^{2}$ The representation is global, since the Fourier series coefficients are obtained from the complete time series. The local character of the waveform is thus defined in terms of global parameters.
}

\section{B. Choosing the Best Waveform Representation}

With the above Hilbert transform in mind, we can now proceed to the estimation of the error related potential waveform given a collection of signal observations $\mathbf{x}(t) \in$ $\mathbb{R}^{N}$. Consider first an instantaneous mixture model, where some underlying latent signals $\mathbf{s}(t) \in \mathbb{R}^{M}$ are related to the observations $\mathbf{x}(t)$ by a constant through time, but unknown linear orthogonal transform $\mathbf{A} \in \mathbb{R}^{N \times M}$, i.e. $\mathbf{x}(t)=\mathbf{A} \mathbf{s}(t)$. We consider that for some given $M \leq N$, the set $\mathbf{S}=\{\mathbf{s}(t)\}_{t}$ explains best the set of observations $\mathbf{X}=\{\mathbf{x}(t)\}_{t}$ with respect to the $L^{2}$-norm. In other words,

$$
(\mathbf{A}, \mathbf{s})=\arg \min _{(\mathbf{Q}, \mathbf{b})} \sum_{t}\|\mathbf{x}(t)-\mathbf{Q b}(t)\|_{2}^{2},
$$

where $\mathbf{Q} \in \mathbb{R}^{N \times M}$, and $\mathbf{Q}^{T} \mathbf{Q}=\mathbf{I}_{M}$, the identity matrix in $\mathbb{R}^{M \times M}$. The solution to the minimization problem of Eq. (2) can be found by taking the singular value decomposition (SVD) of $\mathbf{X}$ as $\mathbf{X}=\mathbf{U} \boldsymbol{\Sigma} \mathbf{V}^{T}$. $\mathbf{A}$ and $\mathbf{S}$ can then be identified with the first $M$ columns of the matrix $\mathbf{U}$, respectively $\mathbf{V} \boldsymbol{\Sigma}^{T}$.

In a similar way, we may calculate the SVD of $\tilde{\mathbf{X}}=$ $\{\tilde{\mathbf{x}}(t)\}_{t}$ as $\tilde{\mathbf{X}}=\tilde{\mathbf{U}} \tilde{\boldsymbol{\Sigma}} \tilde{\mathbf{V}}^{H}$, where the singular values on the diagonal of $\tilde{\boldsymbol{\Sigma}}$ are real ${ }^{3}$. Our set of observed signals $\mathbf{x}(t)$ can thus be approximated as $\mathbf{x}(t)=\Re\{\tilde{\mathbf{x}}\}(t) \approx \Re\{\tilde{\mathbf{A}} \tilde{\mathbf{s}}\}(t)$, where $\tilde{\mathbf{A}}$ and $\{\tilde{\mathbf{s}}(t)\}_{t}$ can be identified with the first $M$ columns of $\tilde{\mathbf{U}}$ and $\tilde{\mathbf{V}} \tilde{\mathbf{\Sigma}}^{T}$, respectively. The set of generating waveforms $\tilde{\mathbf{s}}(t)=\left\{\tilde{s}_{m}(t)\right\}_{m=1}^{M}$ now is composed of analytic signals. In addition to the amplitude scaling found in $\mathbf{A}$, we now also allow for a phase shift of the waveform by allowing for complex entries in $\tilde{\mathbf{A}}$. We may write equivalently

$$
\mathbf{x}(t)=\sum_{m=1}^{N} \Re\left\{\left[\tilde{\mathbf{u}}_{m}\left(\tilde{\sigma}_{m} \tilde{v}_{m}\right)\right](t)\right\}=\sum_{m=1}^{N} \Re\left\{\left[\begin{array}{ll}
\tilde{\mathbf{a}}_{m} & \tilde{s}_{m}
\end{array}\right](t)\right\}
$$

where $\tilde{\mathbf{a}}_{m}$ is the $m$-th column of $\tilde{\mathbf{A}}$. Taking the influence of $\tilde{s}_{m}(t)$ on $\tilde{x}_{n}(t)$, we then find that

$$
\tilde{x}_{n}(t)=\left|\tilde{a}_{n m}\right|\left|\tilde{s}_{m}(t)\right| e^{-\imath\left(\psi(t)+\angle \tilde{a}_{n m}\right)},
$$

which corresponds to an amplitude scaling and a phase shift of $\tilde{s}_{m}(t)$

The reconstruction of the observed signal is perfect, i.e., the equality holds in Eq. (3), since for $M=N$

$$
\begin{aligned}
\tilde{\mathbf{x}}(t) & =\mathbf{x}(t)+\imath[\mathcal{H}(x)](t) \\
& =\Re\{\tilde{\mathbf{A}} \tilde{\mathbf{s}}\}(t)+\imath \Im\{\tilde{\mathbf{A}} \tilde{\mathbf{s}}\}(t) .
\end{aligned}
$$

Despite the fact that the decomposition of $\tilde{\mathbf{x}}(t)$ is unitary, we no longer have an orthogonal decomposition of the observed signal part $x(t)$. In what follows we will directly illustrate the benefits of this method with respect to the estimation of error related potentials.

${ }^{3}$ The singular values can always be chosen real. If they were not to be real, it suffices to multiply the $m$-th singular value $\sigma_{m}$ by $r_{m}=\sigma_{m}^{\star} /\left|\sigma_{m}\right|$ and multiplying the corresponding columns of $\tilde{\mathbf{U}}$ and $\tilde{\mathbf{V}}$ correspondingly by $\left(r_{m}^{\prime}\right)^{-1}$ and $\left(r_{m}^{\prime \prime}\right)^{-1}$, with $r_{m}^{\prime} r_{m}^{\prime \prime}=r_{m}$. Remark that this gives us an infinity of solutions for $\tilde{\mathbf{U}}$ and $\tilde{\mathbf{V}}$. But this infinite solution set is an equivalence class for the initial problem. As a consequence, we may choose whatever representative. 